\title{
Shear orientation of nematic phases of clay nanosheets: processing of barrier coatings
}

\author{
Maximilian Röhrl, Judith H. Mettke, Sabine Rosenfeldt, Holger Schmalz, Ulrich Mansfeld, Renee L. Timmins, \\ Christoph Habel, Josef Breu $\mathbb{D}^{\mathbb{B}}$, Franz Durst
}

Received: 15 January 2021/Revised: 23 June 2021/ Accepted: 26 June 2021

(C) The Author(s) 2021

\begin{abstract}
When suspensions are exposed to shear forces, the particles may form ordered structures depending on their shapes, concentrations, and the material. For some processes, e.g., for wet-film coating, it is important to know how fast these structures form in shear fields and for how long the structures persist when the shear is relaxed. To obtain information on the particle structure formation and the decay time, the effective viscosity of nematic suspensions of $\mathrm{Na}$ hectorite nanosheets was investigated by rheology employing a cone-plate measurement geometry. The necessary time for the formation textured nematic films could be deduced by carrying out effective viscosity measurements at constant time steps. Information could also be obtained on the lifetime of the platelet textures when shear is relaxed. All this information was employed to identify geometrical requirements for slot dies to produce barrier liners with nanosheet layers oriented parallel to PET substrates. Thereby, we obtained green and simple coatings that are in line with state-of-the-art highperformance materials such as metalized plastic foils in terms of oxygen barrier properties.
\end{abstract}

Supplementary Information The online version contains supplementary material available at https://doi.org/10.1007/s11998021-00535-4.

M. Röhrl, S. Rosenfeldt, H. Schmalz, U. Mansfeld,

R. L. Timmins, C. Habel, J. Breu ( $\square)$

Bavarian Polymer Institute and Department of Chemistry, University of Bayreuth, Universitätsstr. 30, 95447 Bayreuth, Germany

e-mail: josef.breu@uni-bayreuth.de

J. H. Mettke, F. Durst ( $\square)$

FMP Technology GmbH, Am Weichselgarten 34,

91058 Erlangen, Germany

e-mail: f.durst@fmp-technology.com
Keywords Rheology, Shear flow-induced alignment, Slot die coating, Barrier properties, Oxygen permeability

\section{Introduction}

When small particles are added to a fluid and shear is applied, as in a Couette flow, the moving suspensions show effective viscosities of different complexities. The latter depend on the suspension medium, the particle concentration, its size and shape, and also on the hydrophobic or hydrophilic surface properties of the particle material. ${ }^{1}$ For instance, Laun et al. showed that the measured effective viscosities as a function of the applied shear stress of polystyrene-ethyl acrylate latex particles in water depend on the particle concentration and the surface charge characteristics of the latex particles. ${ }^{2,3}$ In his seminal work, Giesekus ${ }^{4,5}$ showed that spherical particles, when exposed to shear flows, arrange themselves in such a way that they form "strings of particles". These particle structures were formed when an aqueous dispersion of particles was "moved by hand" between two glass plates. Since the work of Giesekus, it has been found that the effective viscosity of fluid-particle systems changes in such a way that an increase in structured particle arrangements results in a decrease in the measured effective viscosity. In turn, this means that effective viscosity measurements can be employed to reveal structural changes of suspensions induced by increasing shear rates. In particular, shear thinning indicates major structural changes. Such suspensions still comprise of a fluid with its original viscosity, but the interactions of the fluid with dispersed particles and particle-particle interactions yield forces that are recorded by viscometers as a decrease in the measured momentum of a rheometer. ${ }^{5,6}$ The increase in the regularity of the 
particle arrangements in the suspension decreases the viscosity.

Very persistent particle structures can be expected for suspensions of nanosheets with high aspect ratios. ${ }^{7}$ In particular, when the aspect ratio is high enough at a given concentration of the suspension to hinder rotation of adjacent nanosheets, nematic liquid crystalline phases are formed, ${ }^{8}$ as evidenced by small-angle X-ray scattering (SAXS). This is even the case for dilute aqueous suspensions of negatively charged nanosheets of the synthetic clay sodium fluorohectorite $(\mathrm{NaHec}),{ }^{9}$ where the nanosheets are known to adopt a cofacial arrangement due to strong electrostatic repulsion. For instance at $2.3 \mathrm{vol} \%$ of $\mathrm{NaHec}$, adjacent clay nanosheets with a diameter of $20 \mu \mathrm{m}$ are separated to only $32 \mathrm{~nm}$ and consequently, in this nematic state are confined in a cofacial geometry. Individual domains with cofacial arrangement are, however, randomly oriented (Fig. 1a). ${ }^{8}$

Such high aspect ratio nanosheets are perfectly suited for high-performance polymer nanocomposites as needed for a wide variety of applications, ranging from food packaging all the way to encapsulation of optoelectronic devices like organic photovoltaic and organic light emitting devices. ${ }^{10}$ As pointed out by Cussler's theory of the tortuous path ${ }^{11,12}$ for optimization of the barrier properties, perfect parallel alignment of the impermeable nanosheets on the substrate is crucial. Parallel aligned (textured) nanosheets are the key to achieve low gas permeabilities as the diffusion pathway for a gas permeant is hereby maximized and the tortuosity strongly decreases once nanosheets become tilted with respect to the diffusion direction (Fig. 1b). ${ }^{13}$

Flow-alignment of composite materials has been explored for some time. ${ }^{14}$ In particular for the fabrication of composite films of nanosheet fillers, texturing via shear forces has been successfully applied, ${ }^{15-18}$ although the applied methods are limited to rather low shear rates and difficult to scale up, for instance dip coating. A scalable and universally applicable coating process like slot die coating ensures efficient shear
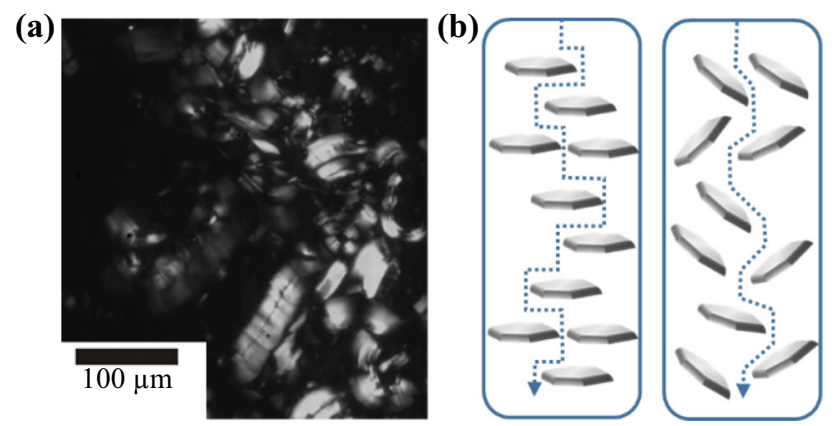

Fig. 1: (a) Optical microscopic image of statistically oriented, swollen NaHec tactoids between crossed polarizers. (b) Scheme of tortuous path for diffusion of permeant depending on the arrangement of nanosheets in a matrix texturing and sufficient inertness with respect to loss of this texture by reorientation to allow freezing of the textured film structure during evaporation of solvent.

Here, we study the shear orientation of nematic $\mathrm{NaHec}$ phases as a function of concentration and shear rate to establish the "formation time" of textured $\mathrm{NaHec}$ suspensions. Moreover, the rheological information was used to lay out slot dies for coating suspensions of $\mathrm{NaHec}$ nanosheets on PET substrates in a single-step procedure and the permeability of these clay-only barriers was determined.

\section{Experimental}

\section{Materials and sample preparation}

Carboxymethyl cellulose (CMC) with a mean molecular weight of $250000 \mathrm{~g} \mathrm{~mol}^{-1}$ was purchased from Daicel Corporation (Japan) and was dissolved in deionized water to obtain a $0.3 \mathrm{wt} \%$ solution. The solution was stable, and no sedimentation appeared. The synthetic clay $\left[\mathrm{Na}_{0.5}\right]^{\text {inter }}\left[\mathrm{Mg}_{2.5} \mathrm{Li}_{0.5}\right]^{\text {oct }}\left[\mathrm{Si}_{4}\right]^{\text {tet }} \mathrm{O}_{10} \mathrm{~F}_{2}$ $(\mathrm{NaHec})$ was synthesized via melt synthesis followed by annealing according to a published procedure and the material featured a cation exchange capacity (CEC) of $1.27 \mathrm{mmol} \mathrm{g}^{-1} \cdot{ }^{19,20}$ Upon immersion in deionized water, the pristine material swells osmotically producing a nematic suspension with delaminated individual nanosheets of an average diameter of $\approx 20 \mu \mathrm{m}$, separated by $32 \mathrm{~nm}$ at $6 \mathrm{wt} \%$, which corresponds to $2.3 \mathrm{vol} \%$. The viscous suspension was placed in an overhead shaker for 7 days to assure equilibration to a uniform swelling state. Less concentrated suspensions were made by diluting this stock suspension with deionized water. A corona-treated PET $(36 \mu \mathrm{m})$ substrate was obtained from Bleher $\mathrm{KG}$ (Germany).

\section{Characterization methods}

Small-angle X-ray scattering (SAXS) was performed using the system "Ganesha Air" (SAXSLAB, Denmark), equipped with a rotating anode X-ray source (copper, MicroMax 007HF, Rigaku Corporation, Japan). A position-sensitive detector (PILATUS 300K, Dectris, Switzerland) was used and different sampleto-detector positions were chosen to cover a wide range of scattering vectors. Measurements of the suspension were performed in $1 \mathrm{~mm}$ glass capillaries (Hilgenberg, Germany) at room temperature. The onedimensional (1D) data $[I(q)$ vs. $q$ with intensity $I$ and scattering vector $q$ ] are in absolute scale and background corrected. The birefringence of the dispersion was evaluated with a self-made crossed polarizer.

Powder X-ray diffraction (PXRD) experiments were performed using $\mathrm{Cu} \mathrm{K} \alpha$ radiation on a BraggBrentano-type diffractometer (Empyrean, Malvern 
Panalytical BV, The Netherlands) equipped with a pixel detector. The XRD pattern of the coated clay film was recorded and subsequently analyzed using Malvern Panalytical's HighScore Plus software. Prior to the measurement, the sample was equilibrated at $43 \%$ relative humidity (r.h.).

All rheological experiments were performed with a shear rheometer (Type Physica MCR 101, Anton Paar, Austria) by using a CP50-1 cone and plate geometry with a plate diameter of $D=50 \mathrm{~mm}$, cone angle of $\beta=1^{\circ}$ and truncation gap of $\kappa=0.102 \mathrm{~mm}$. For determination of the formation time, the viscosity was measured as a function of the time and measuring time steps were set to $0.1 \mathrm{~s}$.

Cryo scanning electron microscopy (CryoSEM) micrographs were recorded at $2 \mathrm{kV}$ with a secondary electron (Everhart-Thornley) detector on a Zeiss Ultra plus SEM equipped with a Leica VCT cryo stage cooled to $-155^{\circ} \mathrm{C}$. For sample preparation, the specimen was sheared in the rheometer directly on the SEM sample holder (Figs. S1, S2) that was subsequently plunged in liquid nitrogen and after cooling was transferred with a Leica EM VCT100 shuttle into a Leica EM MED020 freeze-fracture unit, where fractured surfaces were generated by impacting the specimen with a blade. After sputter coating with a thin layer of tungsten, the sample was transferred with the shuttle to the precooled cryo stage within the SEM for imaging.

Oxygen transmission rates (OTRs) were determined on a Mocon OX-TRAN 2/21 system with a lower detection limit of $0.05 \mathrm{~cm}^{3} \mathrm{~m}^{-2} \mathrm{day}^{-1} \mathrm{~atm}^{-1}$. The measurements were performed at $23^{\circ} \mathrm{C}$ and $50 \%$ and $90 \%$ r.h. A mixture of $98 \%$ nitrogen and $2 \%$ hydrogen $(v / v)$ was used as the carrier gas and pure oxygen as permeant ( $>99.95 \%$, Linde Sauerstoff 3.5).

The thickness of the NaHec barrier coating was measured with a High-Accuracy Digimatic Micrometer (Mitutoyo, Japan) with a measuring range of 0-25 mm and a resolution of $0.1 \mu \mathrm{m}$. A mean value was determined by ten measurements on different spots in the permeability area.

\section{Coating experiments}

An aqueous $6 \mathrm{wt} \% \mathrm{NaHec}$ suspension was used for coating on corona-treated PET foils. The corona treatment guarantees to maintain a uniform wetting of the coated film after the fluid left the slot die. Prior to coating, the suspension was homogenized in a SpeedMixer DAC 400.2 VAC-P (Hauschild, Germany) to remove air bubbles under vacuum (5 min, 50 mbar and up to $2500 \mathrm{rpm}$ ). The coating experiments were conducted using a TSE Table Coater with a 1Layer Slot Die $300 \mathrm{~mm}$, AAA (TSE Troller AG, Switzerland). For the coating procedure, the coating width was set to $0.21 \mathrm{~m}$. The pump flow rate $(3.4 \mathrm{ml}$ $\left.\min ^{-1}\right)$ and the table speed $\left(0.2 \mathrm{~m} \mathrm{~min}^{-1}\right)$ were adjusted accordingly. The table temperature was set to $25^{\circ} \mathrm{C}$ and the coating gap set to $86 \mu \mathrm{m}(36 \mu \mathrm{m}$ PET $+50 \mu \mathrm{m}$ coating height).

The obtained wet films were dried in-line applying a slight under pressure by an airflow of approximately $1.5 \mathrm{~m}^{3} \mathrm{~min}^{-1}$ generated by a Side Channel Blower Type 1SD 510 (Elektror Airsystems GmbH, Germany). A microporous surface below the airflow guarantees a soft and uniformly solvent removal over the entire film surface.

\section{Results and discussion}

\section{Shear orientation of NaHec nanosheets}

Viscosity measurements are usually carried out using a plate-cone rotating viscometer to obtain the dynamic viscosity $\eta$ of liquids. Plain liquids, comprised of low molecular weight molecules, show Newtonian behavior meaning that shear viscosity remains constant and is independent of shear rate. When, however, high molecular weight polymers are dissolved in water at sufficiently high concentration, e.g., carboxymethyl cellulose (CMC), a shear thinning behavior is observed. According to Mezger, ${ }^{6}$ this is related to the disentanglement of the high molecular weight polymers (Fig. 2 inset). For CMC, shear thinning is perfectly reversible with no hysteresis. When the distribution of $\eta$ is measured with increasing and decreasing shear rate $\dot{\gamma}$, the lines overlap (Fig. 2). This indicates that the timeframe available for each $\eta$ measurement is sufficient to yield the $\eta$ value characteristic for each adjusted $\dot{\gamma}$ selected by the rheometer. Owing to limitations of the measurement technology,

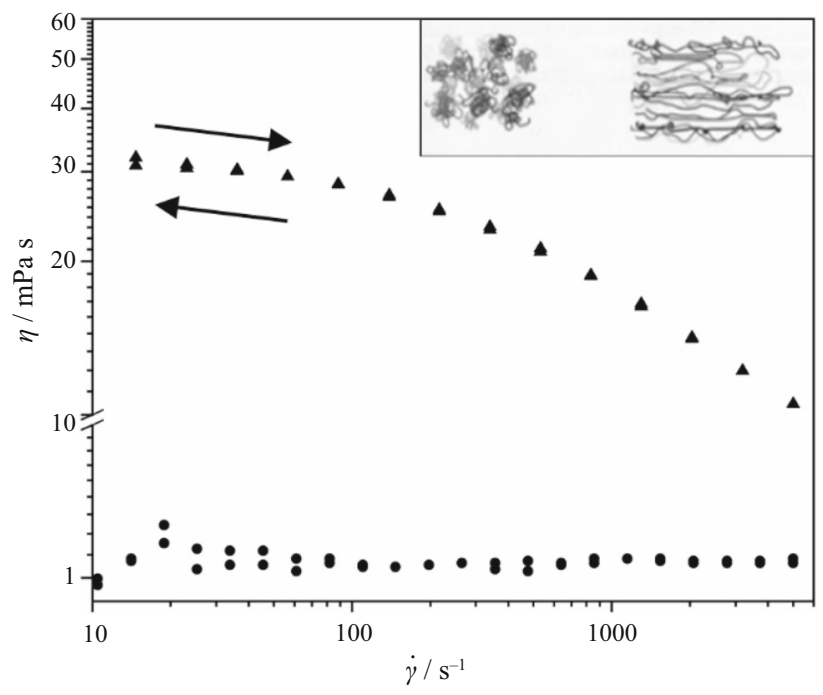

Fig. 2: Viscosity measurements for increasing and decreasing shear rates for water (black circle) and 0.3 wt\% carboxymethyl cellulose solution (black triangle). The black arrows indicate the measurement direction. The inset shows the disentanglement of polymer chains under shear 
the data show a scattering for small shear rates with low-viscosity fluids. Therefore, $\eta$ distributions are shown only for $\dot{\gamma}>10 \mathrm{~s}^{-1}$.

The synthetic clay NaHec belongs to a few layered compounds that show the rare phenomenon of osmotic swelling, ${ }^{21}$ which leads to spontaneous delamination into single $\mathrm{NaHec}$ nanosheets by immersing the clay in deionized water. ${ }^{8}$ A lateral dimension of $20 \mu \mathrm{m}$ with a thickness of $1 \mathrm{~nm}$ for such a nanosheet yields an aspect ratio of $\sim 20000 .{ }^{9}$ Due to this high aspect ratio, nanosheets are hindered in rotation, giving a nematic liquid crystalline phase as evidenced by SAXS (Fig. 3). Even a concentrated ( $6 \mathrm{wt} \%$, corresponding to $2.3 \mathrm{vol} \%$ ) NaHec suspension represents a translationally symmetric, stable, and homogeneous phase owing to the strong electrostatic repulsion of negatively charged clay nanosheets. ${ }^{8}$ Therefore, nanosheets are forced to adopt a cofacial arrangement with single $\mathrm{NaHec}$ nanosheets separated to $32 \mathrm{~nm}$. The SAXS curve shows a $q^{-2}$ dependence at low and intermediate $q$ range that is characteristic for platelet-like objects. Moreover, the osmotic swelling and platelet-like geometry are proven further by employing a simple model of disks with radius $R=10 \mu \mathrm{m}$ and thickness $h=1 \mathrm{~nm}$ separated to $d_{001}=32 \pm 3.2 \mathrm{~nm}$ using a Gauss distribution (Fig. 3 inset).

The inset in Fig. 4a shows schematically the behavior of nanosheets in a fluid under shear, according to Mezger. ${ }^{6}$ Please note that contrary to what was proposed by Mezger, NaHec suspensions used here are not isotropic but due to the large diameter, the nanosheets are held in a coplanar arrangement within domains, as evidenced in the SAXS measurements (Fig. 3). The individual domains, however, are statistically oriented. When shear stress is applied, these

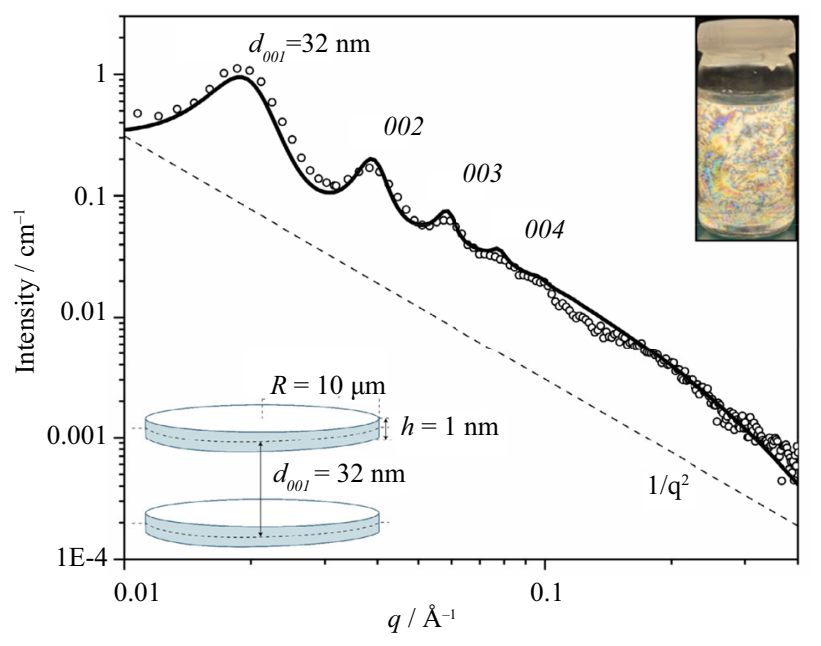

Fig. 3: SAXS pattern of a $6 \mathrm{wt} \% \mathrm{NaHec}$ suspension indicating a nematic phase with a uniform separation of $32 \mathrm{~nm}$ between adjacent nanosheets (circle, measured, black line, calculated). The insets show birefringence of the diluted ( $\sim 1$ wt $\%$ ) nematic suspension between crossed polarizers and the model (separated disks) used for fitting domains are gradually oriented resulting in a quasisingle crystalline state, where all nanosheets are rearranged into a cofacial orientation while still being separated to a uniform distance. With increasing shear rate, the suspension will then eventually represent a Bragg stack (1D single crystal) that is perfectly textured parallel to the rheometer plate (Fig. 4a).

Viscosity-shear rate distributions obtained for NaHec suspensions of varying concentration with increasing and decreasing $\dot{\gamma}$ measurements showed shear thinning and a pronounced hysteresis (Fig. 4a). As expected, the initial viscosity decreased significantly with decreasing concentration. When shear is relaxed, the textures of cofacially aligned $\mathrm{NaHec}$ nanosheets, generated by the velocity shear field of the suspension, persist for a long time although the texture-producing shear field is reduced. Hereby, the measured viscosities stay constant though the shear stress is decreased confirming the high stability of textures of shear oriented $\mathrm{NaHec}$ nanosheets. This phenomenon is more pronounced for suspensions of lower NaHec concentrations. As the dominating interparticle force is electrostatic repulsion, ${ }^{22}$ which increases with $r^{-1}$, this observation is somewhat counterintuitive. If shear thinning is related to the alignment of domains and individual nanosheets in the direction of the shear force, the increase in $\eta$ with decreasing shear rate should be related with tilting and potentially undulating nanosheets in respect to the shear flow. This should actually be more difficult at higher repulsion.

Shear alignment of nanosheets in the suspension was additionally visualized by directly taking CryoSEM images of vitrified suspension films. To suppress crystallization of water that would destroy the pristine suspension structures, for this experiment, the concentrations had to be increased to $20 \mathrm{wt} \%$ and additionally $30 \%$ of glycerol had to be added (see the supporting information for experimental details, Figs. $\mathrm{S} 1, \mathrm{~S} 2$ ). After applying shear stress (up to $1000 \mathrm{~s}^{-1}$ ) while the plate was replaced with a SEM sample holder for the NaHec suspension, it was vitrified and subsequently CryoSEM micrographs were recorded at freeze-fractured surfaces (Fig. 4b, c). As expected based on the rheology experiments, band-like structures with parallel plates translocated relative to each other were the dominating feature.

To investigate the formation time $\Delta t_{\mathrm{f}}$ of oriented nematic phases with nanosheets aligned in parallel to the plate and cofacially to each other, three different but constant shear rates for the rheometer were set and the effective viscosity of a $1.5 \mathrm{wt} \% \mathrm{NaHec}$ suspension was measured over a period of time (Fig. 5). During the formation time, the instantaneously applied shear is responsible for the imposed forces acting on the nanosheets that cause the nanosheets to orientate towards the direction of the flow lines. Hereby, the effective viscosity varies and finds a minimum once the nanosheets aligned themselves in parallel to the walls of the rotating plate of the rheometer. After approximately twice the formation time, the nanosheets 


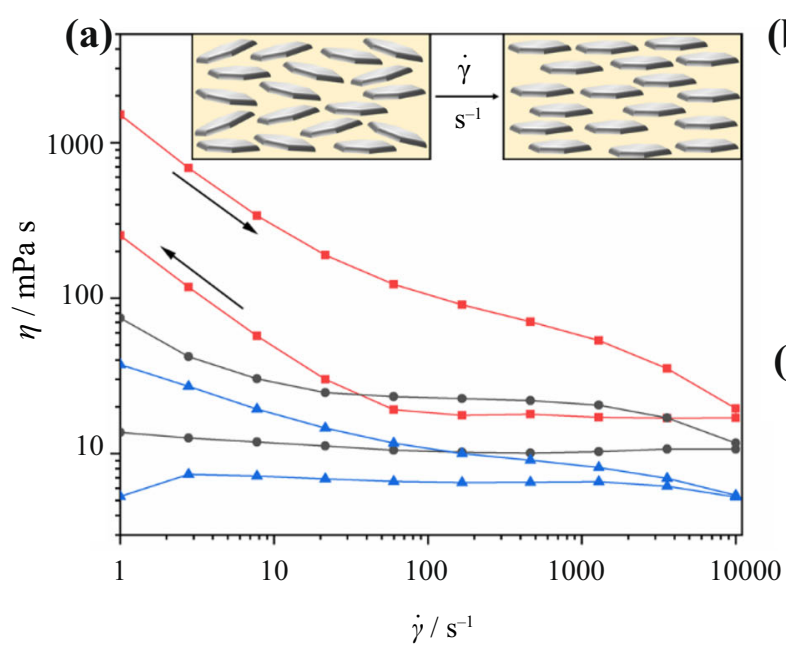

(b)

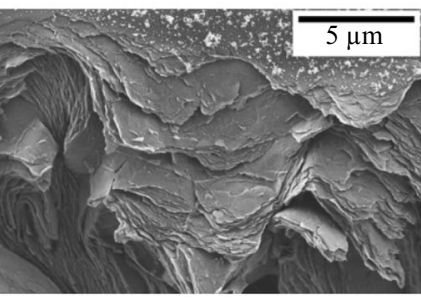

(c)

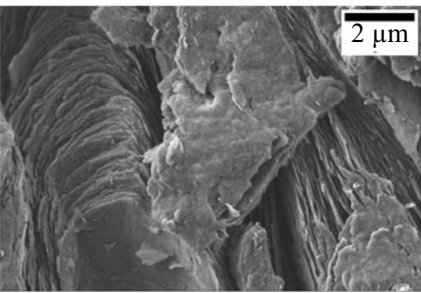

Fig. 4: (a) Effective viscosity measurements of NaHec suspensions of different concentrations: 3 wt $\%$ (red), 1.5 wt\% (gray) and $0.75 \mathrm{wt} \%$ (blue). The black arrows indicate the measurement direction, which is identical for each concentration. The inset shows schematically the behavior of nanosheets under shear. (b), (c) CryoSEM images of shear oriented NaHec suspensions $(20 \mathrm{wt} \%)$ in a water/glycerol $70 / 30 \mathrm{w} / \mathrm{w}$ mixture

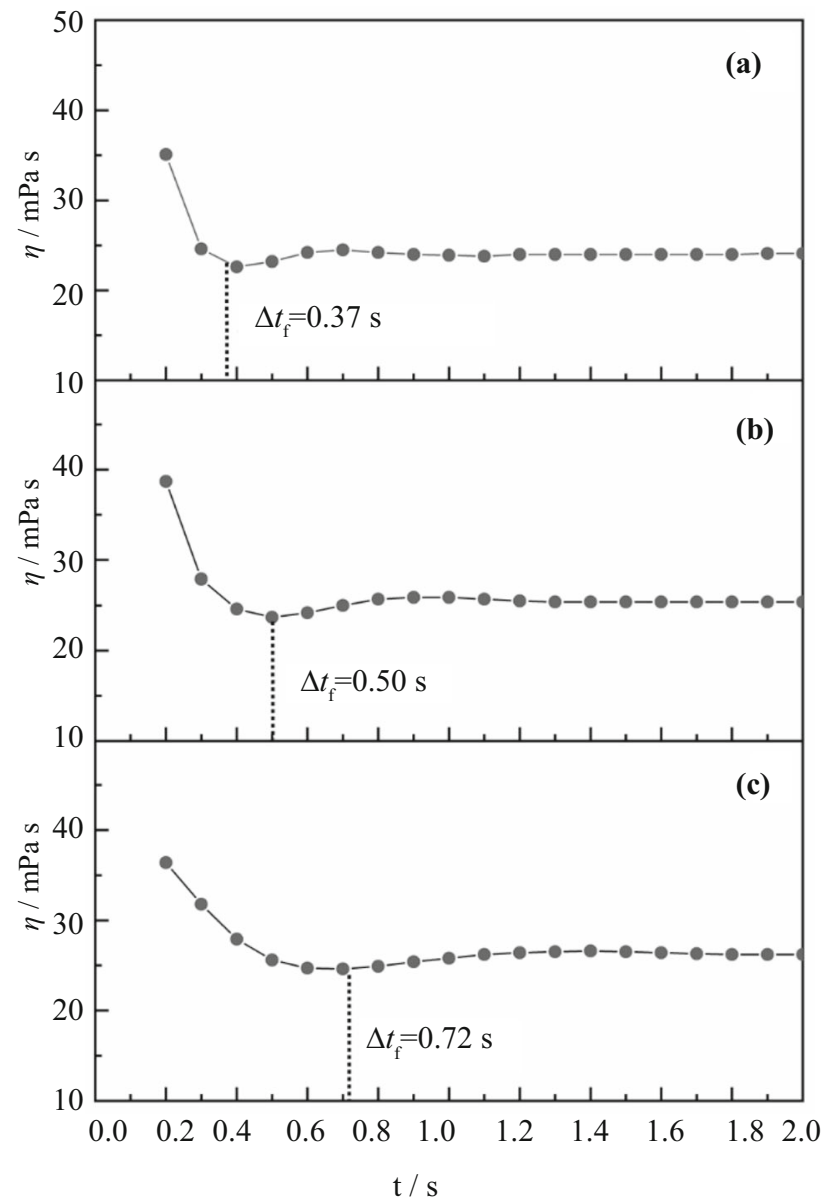

Fig. 5: Measured formation time $\Delta t_{\mathrm{f}}$ of oriented nematic phases in a $1.5 \mathrm{wt} \% \mathrm{NaHec}$ suspension for different shear rates: (a) $100 \mathrm{~s}^{-1}$; (b) $70 \mathrm{~s}^{-1}$; (c) $50 \mathrm{~s}^{-1}$ moved back into their final position, and the viscosity reaches a constant value characteristic of the textured nematic phase. While $\eta$ converges to the same value within experimental errors, the formation times increase with decreasing constant shear field $\dot{\gamma}$ applied. The limiting value of $\eta$ is characteristic for a given concentration (Fig. S3) indicating that the quality of texture approached is the same, although it takes longer to achieve it.

From these measurements, a formation time of the shear oriented texture can be deduced that corresponds to the minimum of the viscosity. Multiplying $\Delta t_{\mathrm{f}}$ with each shear rate applied yields the following relation:

$\Delta t_{\mathrm{f}}=\frac{36}{\dot{\gamma}}$

Hence, the stronger the applied shear field is, the faster the aligned nematic phase forms. The constant 36 was determined as a mean value of the three measurements. So far, our results indicate that effective shear viscosity measurements permit information to be obtained about texturing of nanosheet suspensions. Next, this information will be transformed into scalable processing of barrier films that require such a texture.

\section{Slot die coating}

One of the major applications of nanosheets is barrier coatings applied in a technically benign processing on substrates. Since the viscosity of nanosheet suspensions is considerable, the concentrations that can be processed are low (Fig. 4). Shear thinning helps but since evaporation of large amounts of suspension medium 
takes time, sufficient inertness of the shear alignment is required. The observed rheological behavior of $\mathrm{NaHec}$ suspensions suggests that high shear fields should be employed to yield aligned nanosheets parallel to the substrate already within the applied coating tool. Slot dies of the kind illustrated in Fig. 6 promise to be advantageous for this task. The suspension enters the slot die via the fluid supply slot. The actual coating region is located between the lips $l$ of the die and the substrate to be coated, that moves with a certain web velocity $U_{\mathrm{W}}$. The flow regions underneath the lips possess the shear field of a Couette flow and, if this flow dominates, a nanosheet suspension is exposed to this shear field for the following experimental time $\Delta t_{\mathrm{ex}}$ :

$\Delta t_{\mathrm{ex}}=\frac{3 l}{U_{\mathrm{w}}}$

After the suspension leaves the fluid supply slot (Fig. 6), it moves first to the direction of the back meniscus and then passes two more times the lip length $l$ before it leaves the shear field between the lips and the substrate at the front meniscus. This yields three times the length of the lips $l$ as given in equation (1), assuming the slot width is negligible. By including the coating gap $d$, the actual shear rate $\dot{\gamma}_{\text {ex }}$ to which the suspension is exposed can be calculated as follows:

$\dot{\gamma}_{\mathrm{ex}}=\frac{U_{\mathrm{w}}}{d}$

Taking the formation time $\Delta t_{\mathrm{f}}$ of the nanosheet textures into account, i.e., equation (1), allows the following time consideration to be written:

$\Delta t_{\mathrm{ex}} \geq \Delta t_{\mathrm{f}}=\frac{36}{\dot{\gamma}}=36 \frac{d}{U_{\mathrm{w}}}$
If this time condition is maintained, the nanosheet suspension is exposed for a sufficiently long time to form the shear oriented structures that were seen using the Anton Paar rheometer. Combining equations (2) and (4) yields

$\frac{3 l}{U_{\mathrm{w}}} \geq 36 \frac{d}{U_{\mathrm{w}}}$

$\frac{l}{12} \geq d$

Hence, the rheological measurements combined with the above considerations give access to the geometry of slot dies needed to achieve sufficient nanosheet alignment in the coating process. If one decides to employ a slot die in its "bead coating" mode, the following condition ${ }^{23,24}$ must hold:

$d \geq 2 h$

where $h$ is the coated layer thickness (Fig. 6) and hence equations (6) and (7) can be combined to give

$l \geq 24 h$

Coating textured films of nanosheets require slot dies with "long lips" to be employed, as equation (8) shows. Alternatively, a slow web velocity $U_{\mathrm{w}}$ can be applied for the coating procedure that increases the experimental time $\Delta t_{\mathrm{ex}}$, as equation (4) suggests. In summary, increasing the lip length $l$ and decreasing $U_{\mathrm{w}}$ both will increase $\Delta t_{\mathrm{ex}}$, and the combination will ideally produce highly textured films. We emphasize that this implies that rheological measurements that yield effective viscosities of nanoparticle suspensions provide useful information for the design of coating tools.

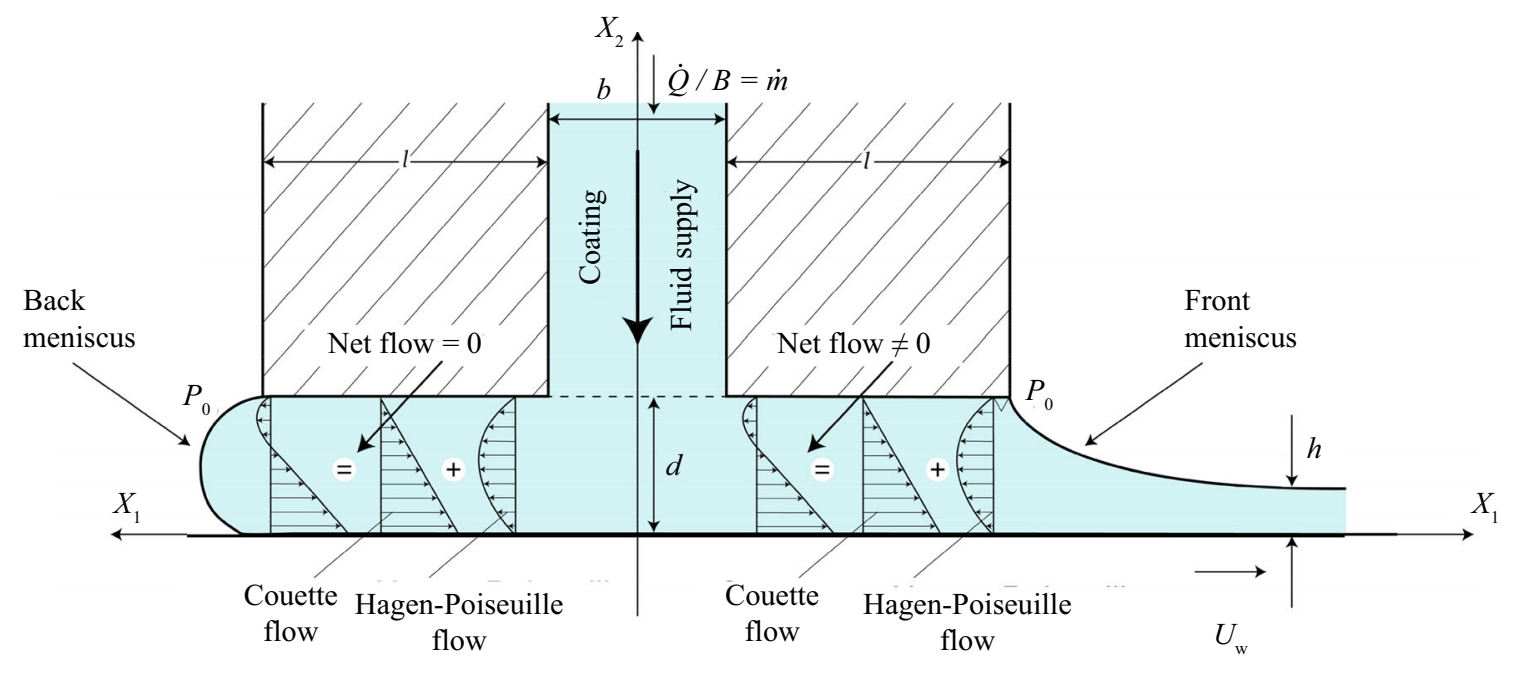

Fig. 6: Schematic representation of the coating flow of a slot die intended for coating flow considerations. The scheme was redrawn according to Dongari et al. ${ }^{23}$ 
To coat nanosheet suspensions appropriately to produce foils with good barrier layers, it is essential to choose a suitable slot die design for the requested layer thickness $h$, according to equation (8). We employed a slot die with lip length $l=600 \mu \mathrm{m}$ allowing to coat layers of $h \leq 25 \mu \mathrm{m}$. Therefore, the requirements discussed above were fulfilled. The coating and drying process was performed using the equipment shown in Fig. 7 that can easily be adapted to large scale and high throughput at an industrial level. A NaHec suspension $(6 \mathrm{wt} \%)$ was coated onto a corona pretreated PET foil with a coating gap of $50 \mu \mathrm{m}$ yielding a wet film thickness of approximately $25 \mu \mathrm{m}$. In addition, a slow web velocity $U_{\mathrm{w}}$ of $0.2 \mathrm{~m} \mathrm{~min}^{-1}$ was applied to guarantee sufficiently experimental time $\Delta t_{\mathrm{ex}}$ for the formation of aligned nanosheet textures in parallel to the substrate already within the shear field of the slot die. In this way, a $1.5 \mu \mathrm{m}$ thick clay-only barrier coating consisting only of overlapping $\mathrm{NaHec}$ nanosheets was obtained as visualized in the SEM image (Fig. S4). Apparently, this hints at the sufficient inertness of the shear oriented textures upon drying and solvent removal. The NaHec nanosheets are perfectly aligned with respect to the PET substrate.

The quality of texture of the $\mathrm{NaHec}$ coating was investigated by 2D SAXS measurements (Fig. 8a). The sample was oriented parallel to the beam direction. The weak halos along the $q_{y}$-axis are characteristic of the PET substrate and appear vertically to the beam direction (compare Fig. S5). In parallel with the PET domains, 001 Bragg reflections of the 1D crystalline $\mathrm{NaHec}$ films are visible along the $q_{y}$-axis, indicating parallel arrangement of the $\mathrm{NaHec}$ nanosheets relative to the PET substrate. The absence of other peaks in the 2D SAXS pattern proves that the clay nanosheets are aligned perfectly parallel and no other orientation is present. For comparison, the inset at the bottom half shows the theoretical scattering of perfectly aligned disks $\left(R=10 \mu \mathrm{m}, h=1 \mathrm{~nm}, d_{001}=1.3 \mathrm{~nm}\right)$. The SAXS data thus confirm that a perfect texture was achieved

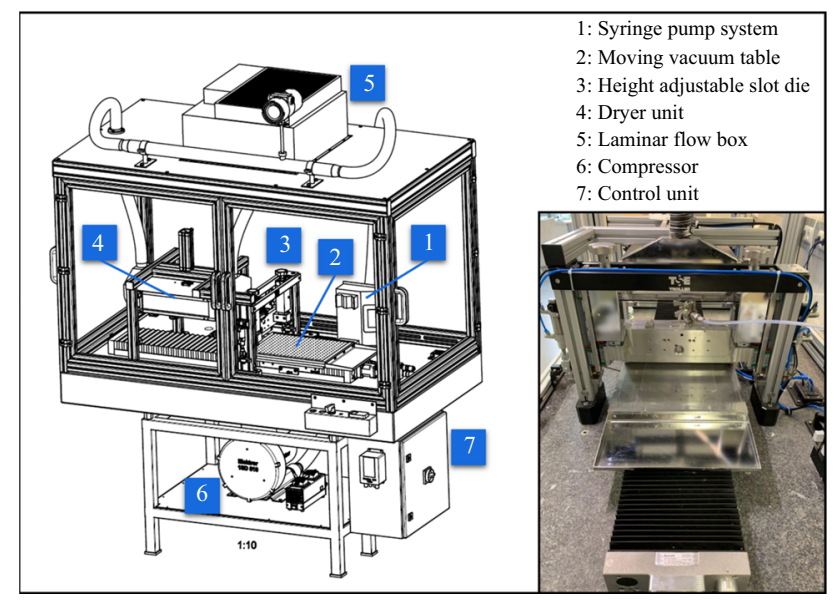

Fig. 7: Sketch of the coating and drying facility. The inset shows the operating slot die (TSE Troller AG) by shear orientation below the slot die during the coating process, and moreover, that this texture is preserved upon drying of the wet film.

Furthermore, the $\mathrm{NaHec}$ film was equilibrated at $43 \%$ r.h. and subsequently analyzed by powder X-ray diffraction (PXRD). A series of basal reflections was visible up to fourth order with a low coefficient of variation (CV) of $0.1 \%$ (Fig. $8 \mathrm{~b}$ ), which according to Meuring's rules ${ }^{25}$ indicates a perfect $1 \mathrm{D}$ crystallinity with constant separation of adjacent nanosheets. The broad peak at $26^{\circ} 2 \theta$ marked with an asterisk arises from the PET substrate (Fig. S6). The basal spacing of $12.6 \AA$, moreover, agrees within experimental error with the value observed for pristine, as synthesized NaHec. ${ }^{19}$ This together with the low CV indicates that upon drying a perfect 1D "single crystalline" film was formed by restacking cofacially aligned nanosheets into a perfectly textured Bragg stack oriented parallel to the PET foil as already indicated by the SAXS data. Faults in the $1 \mathrm{D}$ periodicity caused by misalignment or inclusion of crumbled or folded nanosheets or larger voids could not be detected. This is further corroborated by the high transparency of the $\mathrm{NaHec}$ coating (Fig. 8b inset), as larger voids would cause scattering.

According to the tortuous path mode $1^{11,12}$ and in addition to the aspect ratio of the filler, the quality of texture plays an important role in achieving superior gas barrier properties, and the perfect parallel alignment of nanosheets is expected to produce films with low permeability. And indeed, the clay-only films produced by slot die coating showed oxygen transmission rates (OTRs) comparable to vacuum-deposited aluminum coatings (Table 1$){ }^{26}$ All OTRs were measured at $23^{\circ} \mathrm{C}$ and equilibration to the applied r.h. was guaranteed.

The OTR of neat PET could be decreased by a factor of more than 20 at $50 \%$ r.h. and $23^{\circ} \mathrm{C}$ by applying this thin coating $(1.5 \mu \mathrm{m})$ of NaHec. Absolute transmission values are relevant for industrial applications, but they depend strongly on the thickness of the investigated barrier liner. For better comparison with other materials, transmission rates were converted into permeabilities by multiplying with the overall thickness of the coated PET foil. Moreover, the contribution of the PET substrate to the total OTR is subtracted by applying a series expansion according to Roberts et $\mathrm{al}^{27}$ Thereby, an oxygen permeability (OP) of $2.2 \mathrm{~cm}^{3} \mu \mathrm{m} \mathrm{m}^{-2}$ day $^{-1}$ bar $^{-1}$ was obtained for the neat $\mathrm{NaHec}$ coating, which surpasses that of commonly used polymer materials in the packaging segment by orders of magnitude. For instance, the OP for PET applied as substrate is $1321 \mathrm{~cm}^{3} \mu \mathrm{m} \mathrm{m}^{-2}$ day $^{-1} \mathrm{bar}^{-1}$ and that for poly(vinyl chloride) is $\sim 2000-8000 \mathrm{~cm}^{3} \mu \mathrm{m} \mathrm{m}^{-2}$ day $^{-1} \mathrm{bar}^{-1} \quad\left(2-8 \mathrm{~cm}^{3} \mathrm{~mm} \mathrm{~m}^{-2}\right.$ day $^{-1} \mathrm{bar}^{-1}$, respectively) ${ }^{26}$ at $23^{\circ} \mathrm{C}$ and $50 \%$ r.h. Quite surprisingly and despite the known increased swelling of $\mathrm{NaHec}$ at elevated r.h., ${ }^{8}$ the $\mathrm{OP}$ of the $\mathrm{NaHec}$ coating at $23^{\circ} \mathrm{C}$ and $90 \%$ r.h. deteriorates by only $80 \%$. The observed OP of $3.9 \mathrm{~cm}^{3} \mu \mathrm{m} \mathrm{m}^{-2}$ day $^{-1} \mathrm{bar}^{-1}$ at this r.h. indicates a lower moisture sensitivity as hydrophobized 
(a)

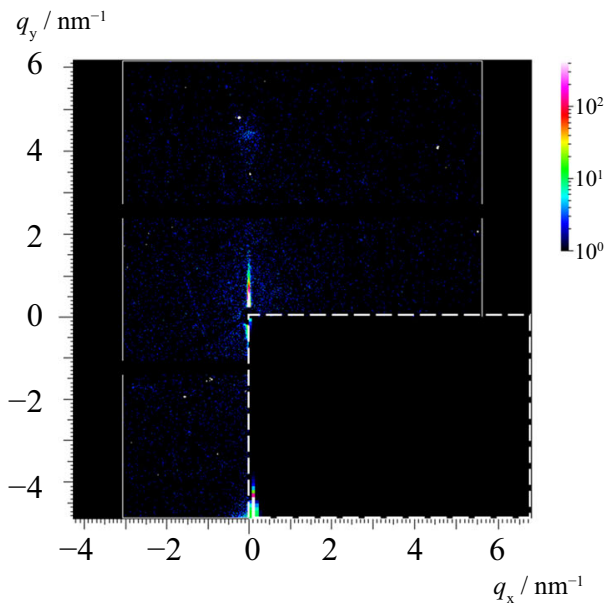

(b)

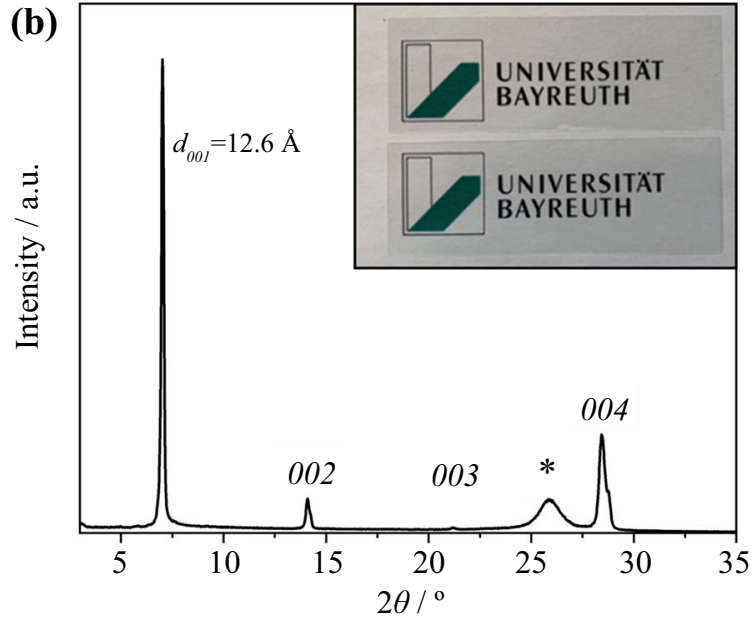

Fig. 8: (a) 2D SAXS pattern of NaHec films on PET measured in parallel geometry. The inset at the bottom half shows the theoretical pattern with a 001 Bragg reflection at $1.3 \mathrm{~nm}$ (b) PXRD pattern of NaHec coating. The inset shows transparent PET (top) and NaHec (bottom) films

Table 1: OTRs of slot die coated NaHec films $(1.5 \mu \mathrm{m})$ measured at $23^{\circ} \mathrm{C}$ and different r.h.

Film

\begin{tabular}{cc} 
OTR $/ \mathrm{cm}^{3} \mathrm{~m}^{-2}$ day $^{-1}$ bar $^{-1}$ \\
\hline $23^{\circ} \mathrm{C}, 50 \%$ r.h. & $23^{\circ} \mathrm{C}, 90 \%$ r.h. \\
\hline 36.7 & 33.0 \\
1.4 & 2.4
\end{tabular}

poly(ethylene-co-vinyl alcohol) (EVOH). An EVOH type with an ethylene content of $27 \mathrm{~mol} \%$, typically used for food packaging, shows an OP deterioration by a factor of $\sim 85$ in the range between 50 and $90 \%$ r.h. ${ }^{28}$

Comparing our results with previously published, clearly inferior OP of synthetic LiHec coatings obtained by doctor blading on polypropylene substrate $\left(60 \mathrm{~cm}^{3} \mu \mathrm{m} \mathrm{m}^{-2}\right.$ day $^{-1}$ bar $^{-1}$ at $23^{\circ} \mathrm{C}$ and $50 \%$ r.h. $){ }^{29}$ would indicate that optimized processing by appropriate slot die coating makes a major contribution to the excellent barrier performance.

Inorganic $\mathrm{NaHec}$ clay-only coatings in combination with the studied slot die coating process are a green and smart solution to producing high oxygen barrier layers that provide stable protection towards gas permeation at elevated r.h.

\section{Conclusions}

Viscosity-shear rate distributions give valuable insight into the kinetics and the required shear stress for shear orientation of nanosheet suspensions. The formation time of these textures is inversely proportional to the applied shear rate in a plate-cone rheometer. With this information, a relation could be deduced that in turn allows for designing slot dies that assure shear orien- tation of nematic nanosheet suspensions within the geometry of the slot die. Applying such a slot die to high aspect ratio $\mathrm{NaHec}$ nanosheets suspensions, perfectly aligned 1D "single crystalline" wet films can be obtained with sufficient inertness to maintain their orientation for a long enough time allowing to obtain textured dry films. Slot die coating, therefore, represents a technically benign coating process appropriate to obtain perfectly textured nanosheet films as required for barrier coatings.

Acknowledgments The group at the University of Bayreuth gratefully thank Florian Puchtler for synthesizing the employed clay and Harald Döll of TSE Troller AG for initial training on the slot die coater. We appreciate the support of the Keylab for Optical and Electron Microscopy of the Bavarian Polymer Institute (BPI). Furthermore, we are grateful for images of the slot die coater provided by TSE Troller AG and thank Olena Khoruzhenko for designing graphics. The work at FMP Technology received financial support from the " $2+2$ "'-BMBFCooperation Project NEEDS and also from FMP, which is gratefully acknowledged. This work was supported by the Deutsche Forschungsgemeinschaft (SFB 1357, C2).

Funding Open Access funding enabled and organized by Projekt DEAL.

Conflict of interest The authors declare that they have no conflicts of interest.

Open Access This article is licensed under a Creative Commons Attribution 4.0 International License, which permits use, sharing, adaptation, distribution and reproduction in any medium or format, as long as you 
give appropriate credit to the original author(s) and the source, provide a link to the Creative Commons licence, and indicate if changes were made. The images or other third party material in this article are included in the article's Creative Commons licence, unless indicated otherwise in a credit line to the material. If material is not included in the article's Creative Commons licence and your intended use is not permitted by statutory regulation or exceeds the permitted use, you will need to obtain permission directly from the copyright holder. To view a copy of this licence, visit http://creativecommons.org/licenses/by/4.0/.

\section{References}

1. Sharma, AK, Tiwari, AK, Dixit, AR, "Rheological Behaviour of Nanofluids: A Review." Renew. Sustain. Energy Rev., 53 779-791 (2016)

2. Laun, HM, "Rheological Properties of Aqueous Polymer Dispersions." Die Angew. Makromol. Chem., 123 (1) 335359 (1984)

3. Laun, HM, "Rheological Properties of Polymer Dispersions with Respect to Shear-Induced Particle Structures." In: Giesekus, H, Hibberd, MF (ed.) Progress and Trends in Rheology II, pp. 287-290. Steinkopff, Heidelberg (1988)

4. Giesekus, H, "Strömungen Mit Konstantem Geschwindigkeitsgradienten und Die Bewegung von Darin Suspendierten Teilchen." Rheol. Acta, 2 (2) 112-122 (1962)

5. Giesekus, H, Phänomenologische Rheologie: Eine Einführung. Springer, Berlin, Heidelberg (1994)

6. Mezger, TG, Das Rheologie Handbuch: Für Anwender von Rotations- und Oszillations-Rheometern. Vincentz Network GmbH \& Co. KG, Hannover (2016)

7. Litchfield, D, Baird, D, "The Rheology of High Aspect Ratio Nanoparticle Filled Liquids." Rheol. Rev., 1-60 (2006)

8. Rosenfeldt, S, Stöter, M, Schlenk, M, et al. "In-Depth Insights into the Key Steps of Delamination of Charged 2D Nanomaterials." Langmuir, 32 (41) 10582-10588 (2016)

9. Stöter, M, Kunz, DA, Schmidt, M, et al. "Nanoplatelets of Sodium Hectorite Showing Aspect Ratios of $\approx 20000$ and Superior Purity."' Langmuir, 29 (4) 1280-1285 (2013)

10. Ebina, T, "Development of Clay-Based Films." Chem. Rec., 18 (7-8) 1020-1032 (2018)

11. Cussler, EL, Hughes, SE, Ward, WJ, et al. "Barrier Membranes." J. Membr. Sci., 38 (2) 161-174 (1988)

12. DeRocher, JP, Gettelfinger, BT, Wang, J, et al. "Barrier Membranes with Different Sizes of Aligned Flakes." $J$. Membr. Sci., 254 (1) 21-30 (2005)

13. Bharadwaj, RK, "Modeling the Barrier Properties of Polymer-Layered Silicate Nanocomposites." Macromolecules, 34 (26) 9189-9192 (2001)

14. Papathanasiou, TD, Guell, DC, Flow-Induced Alignment in Composite Materials. Elsevier, Amseterdam (1997)
15. Zhao, C, Zhang, P, Zhou, J, et al. "Layered Nanocomposites by Shear-Flow-Induced Alignment of Nanosheets." Nature, 580 (7802) 210-215 (2020)

16. Ding, F, Liu, J, Zeng, S, et al. "Biomimetic Nanocoatings with Exceptional Mechanical, Barrier, And Flame-Retardant Properties From Large-Scale One-Step Coassembly." Sci. $A d v ., 3$ (7) e1701212 (2017)

17. Hong, SI, Lee, JH, Bae, HJ, et al. "Effect of Shear Rate on Structural, Mechanical, and Barrier Properties of Chitosan/ Montmorillonite Nanocomposite Film.' J. Appl. Polym. Sci., 119 (5) 2742-2749 (2011)

18. Tsurko, ES, Feicht, P, Nehm, F, et al. "Large Scale SelfAssembly of Smectic Nanocomposite Films by Doctor Blading versus Spray Coating: Impact of Crystal Quality on Barrier Properties." Macromolecules, 50 (11) 4344-4350 (2017)

19. Breu, J, Seidl, W, Stoll, AJ, et al. "Charge Homogeneity in Synthetic Fluorohectorite.” Chem. Mater., 13 (11) 4213-4220 (2001)

20. Kalo, H, Möller, MW, Ziadeh, M, et al. "Large Scale Melt Synthesis in an Open Crucible of Na-Fluorohectorite with Superb Charge Homogeneity and Particle Size." Appl. Clay Sci., 48 (1) 39-45 (2010)

21. Lerf, A, "Storylines in Intercalation Chemistry." Dalton Trans., 43 (27) 10276-10291 (2014)

22. Daab, M, Eichstaedt, NJ, Habel, C, et al. "Onset of Osmotic Swelling in Highly Charged Clay Minerals." Langmuir, 34 (28) 8215-8222 (2018)

23. Dongari, N, Sambasivam, R, Durst, F, "Slot Coaters Operating in Their Bead Mode." Coating, 40 (12) 10-15 (2007)

24. Durst, F, Haas, R, Raszillier, H, "Stabilität vom Filmen in der Fliesser-Filmbeschichtung." Sonderdr. Coat., 22 (1990)

25. Moore, DM, Reynolds, RC, X-ray Diffraction and the Identification and Analysis of Clay Minerals. Oxford University Press, Oxford (1989)

26. Lange, J, Wyser, Y, "Recent Innovations in Barrier Technologies for Plastic Packaging-A Review." Packag. Technol. Sci., 16 (4) 149-158 (2003)

27. Roberts, AP, Henry, BM, Sutton, AP, et al. "Gas Permeation in Silicon-Oxide/Polymer (SiOx/PET) Barrier Films: Role of the Oxide Lattice, Nano-Defects and Macro-Defects." J. Membr. Sci., 208 (1) 75-88 (2002)

28. GmbH, KE. "EVAL ${ }^{\mathrm{TM}}$ Properties." December 2020]; Available from: http://www.evalevoh.com/en/eval-properties /barrier-to-oxygen/relative-humidity.aspx.

29. Möller, MW, Lunkenbein, T, Kalo, H, et al. "Barrier Properties of Synthetic Clay with a Kilo-Aspect Ratio." Adv. Mater., 22 (46) 5245-5249 (2010)

Publisher's Note Springer Nature remains neutral with regard to jurisdictional claims in published maps and institutional affiliations. 